\title{
Unknown Unknowns in Map Preparation for Historical Atlases
}

\author{
Pavel Seemann ${ }^{\mathrm{a},}$ *, Tomáš Janata ${ }^{\mathrm{b}}$ \\ ${ }^{a}$ CTU in Prague, Faculty of Civil Engineering, Department of Geomatics, Czechia, pavel.seemann@fsv.cvut.cz \\ ${ }^{b}$ CTU in Prague, Faculty of Civil Engineering, Department of Geomatics, Czechia, tomas.janata@fsv.cvut.cz \\ * Corresponding author
}

Keywords: Preparation of Cartographic Work, Interdisciplinary Collaboration, Historical Cartography, Thematic Cartography, Atlas, Best Practices

\begin{abstract}
:
Historical maps, like other thematic maps, have their specifics that need to be taken into account when preparing them. A historical map is compiled by a cartographer and a historian. These are professionals with different education (humanistic and technical) and different approaches to work. This results in different authors' trains of thoughts, which may be a source of mistakes in upcoming historical maps. Hidden errors that none of the experts thinks about because they do not know about them or because they seem to be irrelevant in their point of view, are especially serious. The objective of the article is to present the most common unknown unknowns in the preparation of historical maps which the authors acquired during the design of the Academic Atlas of Czech History, the Czech Historical Atlas and other historical maps.
\end{abstract}

Incorrectly portrayed historical boundaries is the first type of error. If the main topic of a map are historical boundaries in particular, i.e. a political or administrative map is designed, the historian specializing in political affairs usually prepares the documents for the cartographer with great care. But if the historian asks for displaying historical boundaries only as support information for the map readers (e.g. for a migration map), they only specify a general requirement (e.g. borders up to 1938). Sometimes, they also provide a background map with these boundaries, which, however, is often in a too small scale. The cartographer, who is inexperienced in the creation of historical maps, then adopts the underlying map uncritically without asking or searching for more detailed sources (larger scale maps, text description of the boundaries) on their own. Also, the cartographer usually does not have deep knowledge of historical development. Then, the cartographer might miss some territorial change, which leads to omissions in the map. Alternatively, a certain part of the border is depicted to a wrong date. The historian does not alert the cartographer to the error because they consider (from their point of view) this small border deviation to be within the limit of the map language. Or, they have no idea what can be considered generalization for the given map scale and what goes beyond that. For historians, in general, the map is rather an illustration complementing their relevant text. The historian also often does not distinguish maps according to their positional accuracy (i.e. it is generally true that a map with a topographic background, harmonized with thematic elements, is more reliable than a map in the form of "colour spots") and it is sufficient for them if a phenomenon or a territory is described in the legend or in the text accompanying the map (written view). Therefore, the cartographer must notify the historian of inappropriate background sources and ask for better quality ones.

The historian often demands in good faith that the upcoming map will capture several historical boundaries of different years. However, the cartographer must verify each partial state, harmonize it with other boundaries and water features and solve different levels of generalization of the data sources. As the number of boundaries increases, the map key is becoming more complicated (issues of colour and map sign structure resolution, border line overlaps). This leads to a substantial increase in the time needed for the preparation of the map. In addition, more information (boundaries) degrades the readability of the map and the map user may not be able to understand it correctly despite the hard work on 
the cartographer's part. In these cases, it is advisable to explain patiently the principle that less is more or, if there is no other way, divide a complex subject into multiple maps.

The way how to write names of sites and locations (settlements) can also be an unknown unknown for both parties. There are usually no problems with current names of settlements based on the Latin script. More difficult, but manageable, is to agree on a system of romanization of non-Latin-script names (e.g. Arabic to Czech). A problem arises in historical maps. The historian and the cartographer should, after mutual discussion, find out whether they will use current names, historical names or common exonyms. Should historical names be used, it is necessary to determine what system of romanization will be used - whether from the official language for a given place and epoch or otherwise. In the case of the preparation of an atlas, it is necessary to enforce that the proposed system will be used by all authors to ensure the uniformity of the work. The cartographer must also consider the technical limitations of computer fonts, which rarely contain all required glyphs for proper romanization.

The examples of unknown unknowns in historical maps preparation show that the ideal state would be if the mapmaker graduated from both cartography and the appropriate discipline of history or historical geography. However, in Czechia, there is insufficient demand for historical maps so that such an expert could hardly make a living off making historical maps only. Thus, cooperation of historians and cartographers is crucial. Communication, mutual learning, high-quality sources for maps and the willingness to discuss the upcoming map with other experts are the key prerequisites to success in eliminating errors. Enough time to prepare maps is another essential precondition. 